\title{
SIMULTANEOUS EXTENSIONS OF A FAMILY OF LINEAR OPERATORS
}

\author{
Rodica-Mihaela Dăneţ, Marian-Valentin Popescu \\ AND NiCOLETA POPESCU
}

\begin{abstract}
In this paper we study when an arbitrary family of (positive) linear operators, defined on vector subspaces in an ordered vector space $E$ with values in a Dedekind complete ordered vector space $F$, has a (positive) linear simultaneous extension. Some previous results of the first author concerning the existence of a (positive) linear common extension for two (positive) linear operators are generalized in the line of an appropriate theorem of D. Maharam. The results obtained are related to the classical Mazur-Orlicz theorem and its famous consequence, the Hahn-Banach theorem. Some applications of the main results pertaining to convex analysis, functional analysis and vector measure theory are given.
\end{abstract}

Mathematics subject classification (2010): 46A22; Secondary 47B60, 47B65.

Keywords and phrases: Common extension of positive linear operators, simultaneous extension of positive linear operators, sublinear operators, Hahn-Banach theorem, Mazur-Orlicz theorem, Maharam theorem.

\section{REFERENCES}

[1] C. D. Aliprantis and O. Burkinshow, Positive Operators, Academic Press, New York, London, 1985.

[2] R. Cristescu, Ordered Vector Spaces and Linear Operators, Ed. Academiei, Bucureşti, Abacus Press, Tunbridge Wells, 1976.

[3] W. Chojnacki, Sur un théorème de Day, un théorème de Mazur-Orlicz, et une généralisation de quelques théorèmes de Silverman, Colloq. Math. 50 (1986), 256-262.

[4] W. ChojnAcki, Erratum: "Sur un théorème de Day, un théorème de Mazur-Orlicz, et une généralisation de quelques théorèmes de Silverman", [Colloq. Math. 50 (1986), 256-262], Colloq. Math., 63 (1992), 139.

[5] R.-M. DĂNEŢ, Common extensions for linear operators, Marcinkiewicz Centenery Volume, Banach Center Publications, Institute of Mathematics, Polish Academy of Sciences, 95 (2011), 299-316. Doi: 10.4064/bc95-0-17.

[6] N. DĂNeŢ AND R.-M. DĂNeŢ, Existence and Extensions of Positive Linear Operators, Positivity 13 (1) (2009), 89-106, published online April 30, 2008, Doi: 10.1007/s11117-008-2220-0.

[7] D. Maharam, Consistent extensions of linear functionals and of probability measures, in: Proc. Sixth Berkely Symp. on Math. Stat. and Probab. (Berkely, 1970/71), Univ. California Press, 1972, vol II, 127-147.

[8] P. MeYer-Nieberg, Banach Lattices, Springer-Verlag, Berlin, 1991.

[9] S. Mazur And W. ORLicz, Sur les espaces métrique linéaires II, Studia Math. 13 (1953), 137-179.

[10] V. PTAK, On a theorem of Mazur and Orlicz, Studia Math. 15 (1956), 365-366.

[11] F. Riesz, Sur la décomposition des opérations fonctionnelles linéaires, Atti. Cong. Int. dei Mat., Bologna 1928, 3 (1930), 143-148.

[12] K. D. SchmidT, Decomposition and extension of abstract measures in Riesz spaces, Rend. Istit. Math. Univ. Trieste 29 (1998) Suppl., 135-213. 